\title{
Testing Systematics of Gaia DR2 Parallaxes with Empirical Surface Brightness: Color Relations Applied to Eclipsing Binaries
}

\author{
Dariusz Graczyk $^{1,2,3}$ (D) , Grzegorz Pietrzyński ${ }^{2,3}$, Wolfgang Gieren ${ }^{1,2}$ (1D) Jesper Storm ${ }^{4}$ (iD, Nicolas Nardetto ${ }^{5}$,

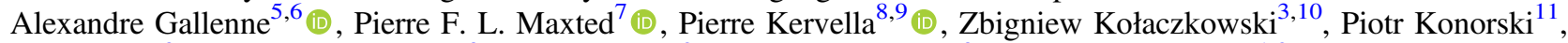 \\ Bogumił Pilecki $^{3}$ (1) , Bartłomiej Zgirski ${ }^{3}$, Marek Górski ${ }^{2}$, Ksenia Suchomska ${ }^{3}$, Paulina Karczmarek ${ }^{1,2}$ (1), Mónica Taormina ${ }^{3}$ (D), \\ Piotr Wielgórski ${ }^{3}$ (1) , Weronika Narloch ${ }^{1,2,3}$, Radosław Smolec ${ }^{3}$ (i), Rolf Chini ${ }^{12,13}$, and Louise Breuval ${ }^{9}$ \\ Millennium Institute of Astrophysics (MAS), Chile \\ ${ }^{2}$ Universidad de Concepción, Departamento de Astronomia, Casilla 160-C, Concepción, Chile; darek@astro-udec.cl \\ ${ }^{3}$ Centrum Astronomiczne im. Mikołaja Kopernika (CAMK), PAN, Bartycka 18, 00-716 Warsaw, Poland; darek@ncac.torun.pl \\ ${ }^{4}$ Leibniz-Institut für Astrophysik Potsdam, An der Sternwarte 16, D-14482 Potsdam, Germany \\ ${ }^{5}$ Université Côte d'Azur, Observatoire de la Côte d'Azur, CNRS, Laboratoire Lagrange, UMR7293, Nice, France \\ ${ }^{6}$ European Southern Observatory, Alonso de Córdova 3107, Casilla 19001, Santiago 19, Chile \\ 7 Astrophysics Group, Keele University, Staffordshire, ST5 5BG, UK

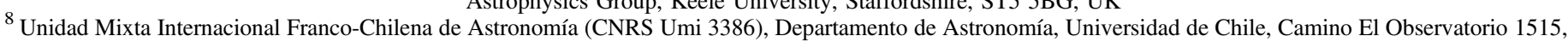 \\ Las Condes, Santiago, Chile \\ ${ }^{9}$ LESIA (UMR 8109), Observatoire de Paris, PSL Research University, CNRS, UPMC, Univ. Paris-Diderot, 5 place Jules Janssen, F-92195 Meudon, France \\ ${ }^{10}$ Instytut Astronomiczny, Uniwersytet Wrocławski, Kopernika 11, 51-622 Wrocław, Poland \\ ${ }^{11}$ Obserwatorium Astronomiczne, Uniwersytet Warszawski, Al. Ujazdowskie 4, 00-478, Warsaw, Poland \\ 12 Ruhr University Bochum, Faculty of Physics and Astronomy, Astronomical Institute, D-44801 Bochum, Germany \\ 13 Instituto de Astronomia, Universidad Catolica del Norte Avenida Angamos 0610, Antofagasta, Chile \\ Received 2018 October 30; revised 2018 December 1; accepted 2018 December 12; published 2019 February 13
}

\begin{abstract}
Using a sample of 81 galactic, detached eclipsing binary stars we investigated the global zero-point shift of their parallaxes with the Gaia Data Release 2 (DR2) parallaxes. The stars in the sample lay in a distance range of 0.04-2 kpc from the Sun. The photometric parallaxes $\varpi_{\text {Phot }}$ of the eclipsing binaries were determined by applying a number of empirical surface brightness-color (SBC) relations calibrated on optical-infrared colors. For each SBC relation we calculated the individual differences $d \varpi_{i}=\left(\varpi_{\text {Gaia }}-\varpi_{\mathrm{Phot}}\right)_{i}$ and then we calculated unweighted and weighted means. As the sample covers the whole sky we interpret the weighted means as the global shifts of the Gaia DR2 parallaxes with respect to our eclipsing binary sample. Depending on the choice of the SBC relation the shifts vary from -0.094 to -0.025 mas. The weighted mean of the zero-point shift from all colors and calibrations used is $d \varpi=-0.054 \pm 0.024$ mas. However, the SBC relations based on $(B-K)$ and $(V-K)$ colors, which are the least reddening dependent and have the lowest intrinsic dispersions, give a zero-point shift of $d \varpi=-0.031 \pm 0.011$ mas in full agreement with results obtained by Lindegren et al. and Arenou et al. Our result confirms the global shift of Gaia DR2 parallaxes of $d \varpi=-0.029$ mas reported by the Gaia team, but we do not confirm the larger zero-point shift reported by a number of follow-up papers.
\end{abstract}

Key words: binaries: eclipsing - parallaxes - stars: distances

Supporting material: machine-readable tables

\section{Introduction}

The Gaia mission (Gaia Collaboration et al. 2016) is a milestone in understanding the Milky Way structure and its chemo-dynamical evolution, but it is also extremely important for the recalibration of standard candles and standard rulers used to construct the extragalactic distance ladder. A number of studies devoted to the recalibration of Cepheids and RR Lyr stars with Gaia Data Release 2 (DR2) parallaxes has already appeared (e.g., Groenewegen 2018; Muraveva et al. 2018).

The possible global systematics of the Gaia DR2 parallaxes were evaluated with different methods by the Gaia consortium. Lindegren et al. (2018) used quasars and internal validation solutions to estimate the systematics and reported that the parallaxes are, on average, about 0.03 mas too small, and that some local significant correlations of parallaxes occur. Arenou et al. (2018) made a comparison of Gaia DR2 parallaxes with a number of external catalogs to evaluate the global shift. Individual shifts vary from $-0.118 \pm 0.003$ mas (HIPPARCOS) to $+0.09 \pm 0.07$ mas (Phoenix dwarf), where the minus sign signifies that Gaia parallaxes are too small. However, most catalogs point to a global systematic offset of about -0.03 mas, e.g., comparisons with the Large and Small Magellanic Cloud star catalogs, which have the smallest formal uncertainties, yield shifts of $-0.0380 \pm 0.0004$ mas and $-0.0268 \pm 0.0006$ mas, respectively.

The global shift was also evaluated by a number of external works. A comparison with distance moduli of 50 Galactic cepheids derived from HST photometry resulted in an estimate of global zero-point offset of Gaia DR2 parallaxes of $-0.046 \pm 0.013$ mas (Riess et al. 2018). Using a sample of 3475 red giant branch stars in the Kepler field from the APOKASC-2 catalog and asteroseismic relations, Zinn et al. (2018) found a systematic shift of $-0.053 \pm 2$ (stat) \pm 1 (syst) mas. The derived shift is specific to the Kepler field but it would also be a measure of the global zero-point shift. Another approach was presented by Stassun \& Torres (2018) who used the eclipsing binary method. They calculated observed reddening-free bolometric fluxes $F_{\text {bol }}$ by fitting the spectral energy distribution and compared them to the bolometric luminosities $L_{\text {bol }}$ to obtain distances. Using a subsample of 89 suitable 
Table 1

Basic Data on the Selected Detached Eclipsing Binaries

\begin{tabular}{|c|c|c|c|c|c|c|c|}
\hline Name & $\begin{array}{c}G A I A \text { DR2 } \\
\text { ID }\end{array}$ & $\begin{array}{l}\text { R.A.(2000) } \\
\text { h:m:s }\end{array}$ & $\begin{array}{l}\operatorname{Decl}_{(2000)} \\
\text { deg:m:s }\end{array}$ & $\begin{array}{c}V \\
(\mathrm{mag})\end{array}$ & $\begin{array}{l}\text { Spectral } \\
\text { Type }\end{array}$ & $\begin{array}{c}\text { Orbital } \\
\text { Period (days) }\end{array}$ & $\begin{array}{c}\text { GAIA DR2 Parallax } \\
\text { (mas) }\end{array}$ \\
\hline MU Cas & 429158427922077184 & $00: 15: 51.560$ & $+60: 25: 53.64$ & $10.808 \pm 0.007$ & $\mathrm{~B} 5 \mathrm{~V}+\mathrm{B} 5 \mathrm{~V}$ & 9.652949 & $0.453 \pm 0.040$ \\
\hline YZ Cas & 539047365205648128 & $00: 45: 39.077$ & $+74: 59: 17.06$ & $5.653 \pm 0.015$ & $\mathrm{~A} 2 \mathrm{~m}+\mathrm{F} 2 \mathrm{~V}$ & 4.4672235 & $10.473 \pm 0.094$ \\
\hline V459 Cas & 522461335380031104 & $01: 11: 29.913$ & $+61: 08: 48.07$ & $10.322 \pm 0.003$ & $\mathrm{~A} 1 \mathrm{~V}+\mathrm{A} 1 \mathrm{~V}$ & 8.4582538 & $1.304 \pm 0.048$ \\
\hline V505 Per & 455772347387763840 & $02: 21: 12.964$ & $+54: 30: 36.28$ & $6.889 \pm 0.016$ & $\mathrm{~F} 5 \mathrm{~V}+\mathrm{F} 5 \mathrm{~V}$ & 4.222020 & $15.970 \pm 0.039$ \\
\hline DN Cas & 513431183821175936 & $02: 23: 11.540$ & $+60: 49: 50.18$ & $9.878 \pm 0.010$ & $\mathrm{O} 8 \mathrm{~V}+\mathrm{B} 0 \mathrm{~V}$ & 2.31095111 & $0.485 \pm 0.032$ \\
\hline
\end{tabular}

(This table is available in its entirety in machine-readable form.)

eclipsing binaries from their catalog (Stassun \& Torres 2016) they derived a global shift of $-0.082 \pm 0.033$ mas. The method used by Stassun \& Torres (2018) is, from a theoretical point of view, the most robust one because it utilizes a very wide range of photometry from UV to midinfrared. However, the $L_{\text {bol }}$ are calculated from the effective temperature of each component and this radiative parameter is almost always the least constrained fundamental parameter of an eclipsing binary. There are two main reasons for this: (1) the wide range of methods used to determine effective temperatures that exhibit significantly different zero-points of the resulting temperature scales, and (2) the notorious difficulty in establishing an absolute temperature scale with a precision better than $1 \%$ (e.g., Casagrande et al. 2014). Thus from a practical point of view the method used by Stassun \& Torres (2018) is not the optimal one because it has some systematics that are still difficult to properly evaluate. The need of an eclipsing binary approach, which would minimize systematic effects and would be based on direct and precise empirical relations, was the prime motivation for this work. As in our previous work (Graczyk et al. 2017, hereafter G17) we focus on an application of surface brightness-color relations.

\section{The Sample}

We extended the sample of 35 eclipsing binaries compiled by G17 by searching for detached systems in the literature suitable for a precise distance determination. In order to make the extension we used catalogs of eclipsing binary systems compiled by a number of authors: Bilir et al. (2008), Torres et al. (2010), Eker et al. (2014), Southworth (2015), and Stassun \& Torres (2016).

We used the same selection criteria as in G17; however, we relaxed the condition on a volume-limited sample $(d<300 \mathrm{pc})$ by also accepting more distant systems. Because the $\beta$ Aur system is too bright to have a Gaia parallax, and AI Phe is a confirmed triple system (M. Konacki 2019, private communication) we drop these systems from our sample. We also included a system from our unpublished work: AL Dor. Following G17 we retained systems with a fractional precision of recent Gaia DR2 parallaxes (Gaia Collaboration et al. 2016, 2018; Lindegren et al. 2018) better then 10\%. A trigonometric parallax $\varpi$ with a fractional error $f=\sigma_{\varpi} / \varpi$ smaller than 0.1 is a good and weakly biased estimator of the true distance (e.g., Bailer-Jones 2015; Bailer-Jones et al. 2018). In our sample only two early and distant systems have $f$ marginally larger than 0.1, i.e., EM Car and DW Car. Most of the sample systems have fractional errors of their parallaxes smaller than 0.06 for which any bias can be completely neglected. Finally our

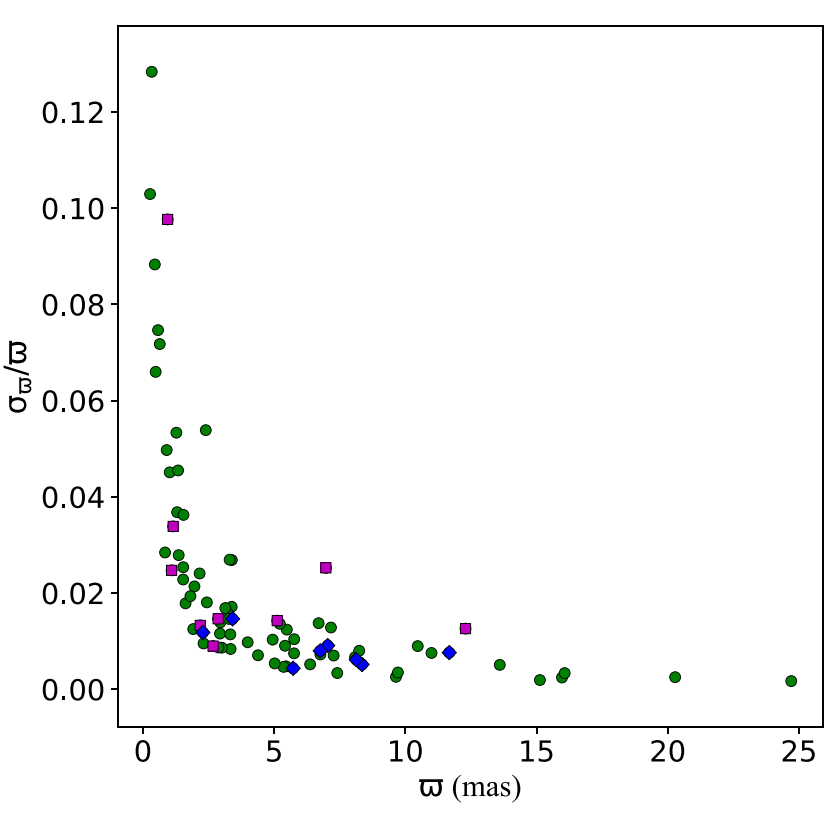

Figure 1. Distribution of fractional error of Gaia DR2 parallaxes for all 81 eclipsing binaries in our sample. Nine systems with suspected binary motion detection or calibration problems are denoted by squares. Eight stars with the significant PMa are denoted by diamonds.

sample contains 81 systems (51 on the northern hemisphere and 30 on the southern one). Their basic parameters are presented in Table 1 and the distribution of the parallax fractional errors is presented in Figure 1.

Arenou et al. (2018) proposed three quality controls to estimate reliability of astrometric solutions of Gaia DR2; they are defined by their Equations (1)-(3). Those quality controls help to filter out most spurious astrometric solutions, but they filter out also some well-defined solutions. In our sample, 9 stars do not fulfill the first criterium (possible double stars, suspected binary motion, or calibration problems) and 7 other stars do not fulfill the third criterium (i.e., visibility_periods_used $<8$ ). However, the third criterium is useful mostly to very crowded fields in the Bulge and as no star in our sample is there, we kept these 7 systems during analysis. The 9 stars that do not fulfill the first criterium are denoted as squares in Figure 1. Only 3 of them have the relative parallax uncertainty larger by a factor of two than expected. We investigated our sample further by looking for the proper motion anomaly (PMa; Kervella et al. 2019), which could be a sign of a photocenter movement or a third body in a system. The PMa results from a comparison of the proper motion 
Table 2

Parameters of the Wilson-Devinney Models

\begin{tabular}{|c|c|c|c|c|c|c|c|c|c|c|c|c|c|c|}
\hline \multirow{3}{*}{$\begin{array}{l}\text { Eclipsing } \\
\text { Binary }\end{array}$} & \multicolumn{9}{|c|}{ Input Parameters } & \multirow{3}{*}{ References } & \multicolumn{4}{|c|}{ WD Model Parameters } \\
\hline & \multicolumn{2}{|c|}{ RV Semiamplitude } & \multicolumn{3}{|c|}{ Orientation of the Orbit } & \multicolumn{2}{|c|}{ Fractional Radius } & \multicolumn{2}{|c|}{ Effective Temperature } & & \multirow{2}{*}{$\begin{array}{c}\text { Semimajor } \\
\text { Axis }\left(R_{\odot}\right)\end{array}$} & \multirow{2}{*}{$\begin{array}{l}\text { Mass } \\
\text { Ratio }\end{array}$} & \multirow{2}{*}{$\Omega_{1}$} & \multirow{2}{*}{$\Omega_{2}$} \\
\hline & $\overline{K_{1}\left(\mathrm{~km} \mathrm{~s}^{-1}\right)}$ & $K_{2}\left(\mathrm{~km} \mathrm{~s}^{-1}\right)$ & $e$ & $\omega(\mathrm{rad})$ & $i(\mathrm{deg})$ & $r_{1}$ & $r_{2}$ & $T_{1}(\mathrm{~K})$ & $T_{2}(\mathrm{~K})$ & & & & & \\
\hline$\overline{\mathrm{MU} \text { Cas }}$ & $107.7(1.0)$ & $105.8(9)$ & $0.1930(3)$ & $0.234(7)$ & $87.02(7)$ & $0.0917(9)$ & $0.1048(10)$ & $15900(500)$ & $15525(500)$ & (1) & 40.0234 & 1.0180 & 12.173 & 10.952 \\
\hline YZ Cas & $73.05(19)$ & $124.78(27)$ & 0.0 & $\ldots$ & $88.33(7)$ & $0.14456(56)$ & $0.07622(33)$ & $9520(120)$ & $6880(240)$ & (2) & 17.4753 & 0.5854 & 7.5141 & 8.8912 \\
\hline V459 Cas & $81.70(60)$ & $83.90(60)$ & $0.0243(4)$ & $4.19(1)$ & $89.47(1)$ & $0.07260(30)$ & $0.07100(30)$ & $9140(300)$ & $9085(300)$ & (3) & 27.6786 & 0.9738 & 14.776 & 14.757 \\
\hline V505 Per & $89.01(8)$ & $90.28(9)$ & 0.0 & $\ldots$ & 87.95(4) & $0.0860(9)$ & $0.0846(9)$ & $6512(21)$ & $6460(30)$ & (4) & 14.9715 & 0.9859 & 12.618 & 12.665 \\
\hline DN Cas & 211(3) & $292(5)$ & 0.0 & $\ldots$ & $77.20(20)$ & $0.3070(20)$ & $0.2460(20)$ & $32100(1000)$ & $28500(1100)$ & (5) & 23.5613 & 0.7226 & 4.0372 & $4.112 \mathrm{C}$ \\
\hline
\end{tabular}

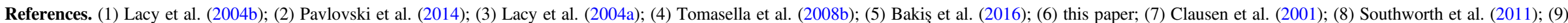

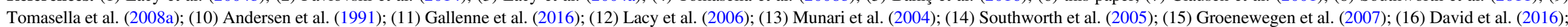

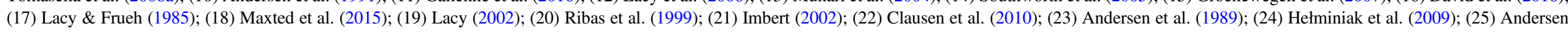

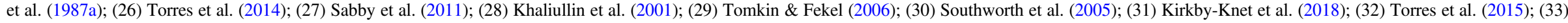

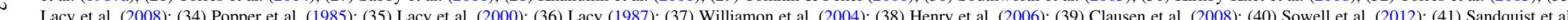

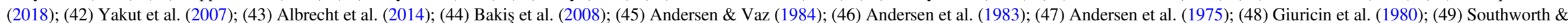

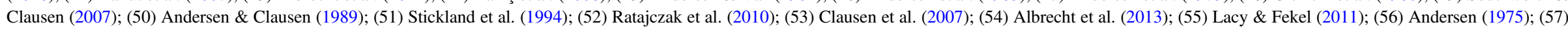

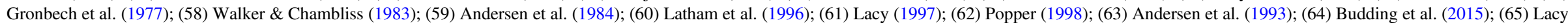

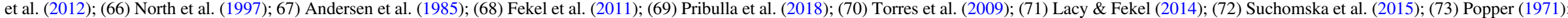

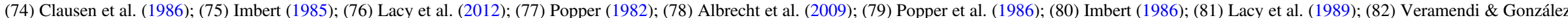

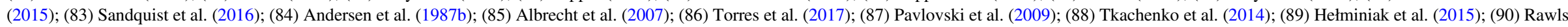

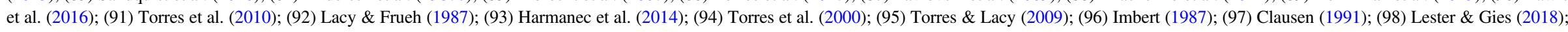

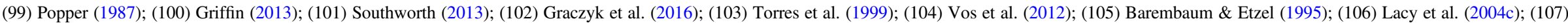
Popper (1983); (108) Demircan et al. (1994); (109) Clausen et al. (2010); (110) Lacy et al. (2014); (111) Çakirli et al. (2009).

(This table is available in its entirety in machine-readable form.) 
Table 3

Corrections to Original 2MASS Magnitudes

\begin{tabular}{lccc}
\hline \hline ID & $K(\mathrm{mag})$ & $H(\mathrm{mag})$ & $J(\mathrm{mag})$ \\
\hline GG Lup & -0.345 & -0.342 & -0.334 \\
KX Cnc & -0.426 & -0.427 & -0.429 \\
WZ Oph & -0.535 & -0.536 & -0.540 \\
YZ Cas & -0.162 & -0.155 & -0.137 \\
BW Aqr & -0.524 & -0.526 & -0.532 \\
SZ Cen & -0.454 & -0.458 & -0.467 \\
V442 Cyg & -0.283 & -0.283 & -0.283 \\
ASAS1800 & -0.512 & -0.505 & -0.493 \\
DW Car & -0.464 & -0.465 & -0.467 \\
\hline
\end{tabular}

vectors at the Hipparcos and Gaia epoch with the mean proper motion computed from a coordinate shift between Hipparcos and Gaia epochs. We found a significant $(\mathrm{SNR}=5)$ detection of the PMa for 8 stars, but interestingly none of them are common with the subsample of 9 suspected stars. The stars with the PMa are denoted as diamonds in Figure 1. All of them have well-defined 5-parametric astrometric solutions, and the $\mathrm{PMa}$, if confirmed with later Gaia data releases, is likely due to long-period changes caused by an unrecognized triple companion.

\subsection{Photometry}

\subsubsection{Optical}

We used Tycho-2 $B_{T}$ and $V_{T}$ photometry (Høg et al. 2000) downloaded from Vizier (Ochsenbein et al. 2000). ${ }^{14}$ The Tycho photometry was subsequently transformed onto the Johnson system using the method outlined by Bessell (2000). Whenever possible we used Johnson $B, V$ photometry from the compilation of Mermilliod (1997) and also absolute optical photometry from literature sources.

\subsubsection{Near Infrared}

We used NIR $J H K_{S}$ photometry of the Two Micron All Sky Survey (2MASS; Skrutskie et al. 2006) from Vizier. ${ }^{15}$ For the purpose of using the SBC relations based on the $(V-K)$ color expressed on the Johnson photometric system we transformed the 2MASS magnitudes using the equations given in Bessell \& Brett (1988) and Carpenter (2001). ${ }^{16}$ The transformation equations are as follows:

$$
\begin{gathered}
K_{J}-K_{2 \mathrm{M}}=0.037-0.017(J-K)_{2 \mathrm{M}}-0.007(V-K)_{2 \mathrm{M}} \\
(J-K)_{J}=1.064(J-K)_{2 \mathrm{M}}+0.006 \\
(H-K)_{J}=1.096(H-K)_{2 \mathrm{M}}-0.027 .
\end{gathered}
$$

\section{Method}

\subsection{Collection of Fundamental Parameters}

For each system we collected orbital and photometric parameters from the literature including the most recent publications. We were searching for basic parameters describing dynamical and geometrical parameters of each system: the radial velocity semiamplitudes $K_{1,2}$, the orbital period $P$, the

\footnotetext{
14 http://vizier.u-strasbg.fr: I/259/tyc2.

15 http://vizier.u-strasbg.fr: II/281/2mass6x.

16 http://www.astro.caltech.edu/ jmc/2mass/v3/transformations /
}

orbital inclination $i$, the eccentricity $e$, the longitude of periastron $\omega$, and the photometric relative radii $r_{1,2}$. These parameters were supported by radiative parameters: the effective temperatures $T_{1,2}$ of both components. The radiative parameters are usually known with the least precision and accuracy. Whenever several papers independently presented the analysis of a given eclipsing binary we adopted the weighted mean for the parameters. However, if there was a significant improvement on the precision of parameters reported in one of papers, then we used only values from that paper. It turned out that we could not always directly trace all the above parameters from literature sources. In some cases when modeling of an eclipsing binary was done with numerical codes based on the Roche formalism (the Wilson-Devinney (WD) code, the ELC code, etc.) we had to calculate relative radii and radial velocity semiamplitudes from the absolute dimensions presented in the relevant papers. The collected parameters are summarized in Table 2.

\subsection{The WD Model of the Systems}

For the purpose of obtaining homogenous parameters for the eclipsing binary sample we decided to create a model of each system following G17. The models were built using the WD code version 2007 (Wilson \& Devinney 1971; Wilson 1979, 1990; van Hamme \& Wilson 2007) while parameters of the models were based on solutions published in the literature (see Section 3.1). All models were checked for internal consistency of the parameters and it turned out that in many cases they had to be fine-tuned. In particular, the temperature ratio and the absolute temperature scale, being important for a precise prediction of infrared light ratios, were inspected carefully.

The procedure was as follows. Dynamical and geometrical parameters were transformed into the semimajor axis of the system $a$, the mass ratio $q$ and into dimensionless Roche potentials $\Omega_{1,2}$ using equations given in Torres et al. (2010) and Wilson (1979). Both $\Omega$ and $q$ are parameters directly fitted or used within the WD program. We usually fixed the temperature of the primary star $T_{1}$ and then, using published light ratios in different photometric bands, we adjusted the temperature of the companion $T_{2}$. In very few cases, however, we also rederived $T_{1}$ using two temperature-color calibrations (Flower 1996; Worthey \& Lee 2011). However the temperature shifts are small and within errors given in the literature. None of the eclipsing binaries in our sample has infrared $J, H, K$ light curves suitable for deriving direct light ratios in those bands. Thus, in order to calculate intrinsic infrared colors of the components of each system we employed eclipsing binary models based on optical light curves and we extrapolated them into the infrared. The extrapolation was done using the atmosphere approximation within the WD code, which uses precomputed intensities based on Kurucz's ATLAS9 models (Kurucz 1993).

The rotation parameter $F_{1,2}$ was kept to 1 (synchronous rotation), unless there was a direct spectroscopic determination of $F$ significantly different from unity. The albedo $A$ and the gravity brightening $g$ were set in a standard way for a convective atmosphere cooler then $7200 \mathrm{~K}$ and radiative ones for a hotter surface temperature. This was done only for the sake of consistency because the two parameters have negligible effect on the light ratios. The input and derived parameters used to create the appropriate WD models are listed in Table 2. 
Table 4

Physical and Photometric Parameters of Individual Components Used to Derive Photometric Parallaxes

\begin{tabular}{|c|c|c|c|c|c|c|c|c|c|c|c|c|c|}
\hline \multirow{2}{*}{$\begin{array}{l}\text { Eclipsing } \\
\text { Binary }\end{array}$} & \multirow{2}{*}{$\begin{array}{c}E(B-V) \\
\quad(\mathrm{mag})\end{array}$} & \multicolumn{2}{|c|}{ Radius $\left(R_{\odot}\right)$} & \multicolumn{5}{|c|}{ Observed Magnitudes } & \multicolumn{5}{|c|}{ Light Ratios } \\
\hline & & $R_{1}$ & $R_{2}$ & $B$ (mag) & $V$ (mag) & $J$ (mag) & $H$ (mag) & $K$ (mag) & $B$ & $V$ & $J$ & $H$ & $K$ \\
\hline MU Cas & $0.478(10)$ & $3.670(43)$ & $4.194(48)$ & $11.112(9)$ & $10.808(7)$ & $10.170(23)$ & $10.092(23)$ & $10.050(16)$ & 1.253 & 1.257 & 1.273 & 1.276 & 1.277 \\
\hline YZ Cas & $0.015(10)$ & $2.526(11)$ & $1.332(6)$ & $5.715(26)$ & $5.660(15)$ & $5.490(20)$ & $5.502(42)$ & $5.475(22)$ & 0.061 & 0.088 & 0.168 & 0.200 & 0.205 \\
\hline V459 Cas & $0.246(24)$ & $2.009(13)$ & $1.965(13)$ & $10.591(9)$ & $10.322(3)$ & $9.791(23)$ & $9.715(25)$ & $9.668(16)$ & 0.936 & 0.941 & 0.949 & 0.950 & 0.951 \\
\hline V505 Per & $0.003(5)$ & $1.288(14)$ & $1.267(14)$ & $7.269(30)$ & $6.846(20)$ & $6.120(70)$ & $5.793(39)$ & $5.795(21)$ & 0.924 & 0.935 & 0.952 & 0.958 & 0.959 \\
\hline DN Cas & $0.917(50)$ & 7.233(96) & $5.796(82)$ & $10.495(18)$ & $9.878(10)$ & $8.413(25)$ & $8.217(19)$ & $8.132(24)$ & 0.492 & 0.500 & 0.521 & 0.527 & 0.529 \\
\hline
\end{tabular}

(This table is available in its entirety in machine-readable form.) 


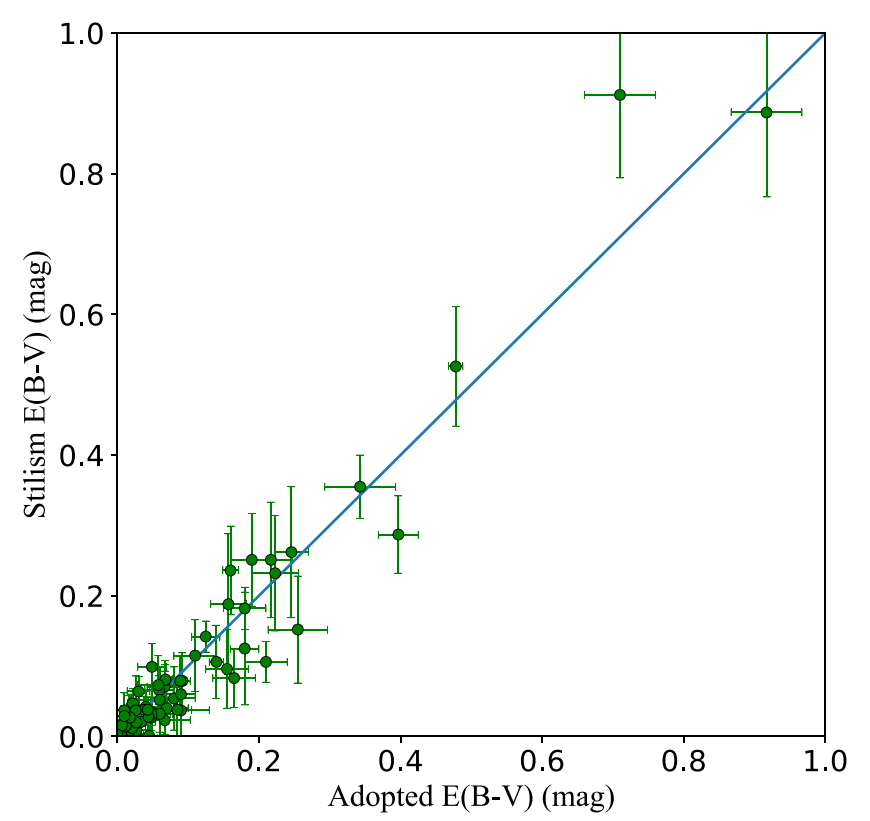

Figure 2. The comparison of our adopted reddening estimates with 3D Stilism extinctions.

\subsection{Correction of 2MASS Magnitudes Taken during Eclipses}

Nine systems from our sample have 2MASS observations taken during the eclipses. To account for the light lost during the minima we used our WD models to calculate the appropriate corrections, which are given in Table 3.

\subsection{Reddening}

Reddenings to each object were taken from the literature. We also derived independently values of $E(B-V)$ using the extinction maps of Schlegel et al. (1998) following the prescription given in Suchomska et al. (2015). Usually as a final value of the extinction we used an average, unless determinations were discrepant or we have at our disposal only one reddening estimate. The adopted reddenings are reported in Table 4. To check them we compared them with independent extinction estimates from 3D extinction maps from Stilism (Lallement et al. 2014; Capitanio et al. 2017)—see Figure 2. On average Stilism extinctions are slightly smaller, but an overall agreement is good, and although the spread is quite large both sets of reddenings are consistent within errors.

\subsection{Intrinsic Magnitudes}

In Table 4 we summarize all parameters used to derive the intrinsic photometric indexes of the component stars. In order to calculate them the photometry was dereddened using the mean Galactic interstellar extinction curve from Fitzpatrick \& Massa (2007) assuming $R_{V}=3.1$. Then the light ratios in the Johnson $B V J H K$ bands were derived using the WD models following G17, and employed to calculate the individual magnitudes and colors. Both the extinction correction and extrapolation of light ratios into the infrared add to uncertainty on derived intrinsic magnitudes. The extinction correction error is given in Table 4 but for the extrapolation uncertainty we did not add additional error. While for systems with similar temperature of components extrapolation leads to negligible additional error in some systems with the large temperature ratio of components this uncertainty may be significant. However when calculating a parallax (or a distance) to a particular eclipsing binary as an average from two components this uncertainty largely cancels out.

\subsection{Photometric Parallaxes}

The eclipsing binary method gives the photometric distance as well as the photometric parallax to a particular target, because both quantities are inferred from observables. Because of this we decided to work in a regime of parallaxes instead of distances to avoid an additional bias, which may arise in the conversion of parallaxes into distances. However, we underline here that working with a regime of parallaxes does not solve the whole problem because, for fractional parallax uncertainties larger then $15 \%-20 \%$, the trigonometric parallax becomes a poor prior on the distance.

\subsubsection{Parallaxes from the Bolometric Flux Scaling}

In order to directly compare our results with those of Stassun \& Torres (2018) we employed the bolometric flux scaling method utilizing the $V$-band bolometric corrections $\mathrm{BC}$ to derive photometric parallaxes. The photometric parallax $\varpi$ to the $i$ th component of the system was calculated using equation:

$$
\varpi_{i}(\operatorname{mas})=2.956 \cdot 10^{10} R_{i}^{-1} T_{i}^{-2} 10^{-0.2\left(\mathrm{BC}_{i}+V_{i}\right)}
$$

where index $i=\{1,2\}, R$ is the radius of a component expressed in solar radii, $T$ is its effective temperature, BC is a bolometric correction interpolated from the Flower (1996) tables for a given temperature and $V$ is the intrinsic magnitude of a component. The parallax to a particular system was calculated as the unweighted average parallax of the two components.

\subsubsection{Parallaxes from the SBC Relations}

The eclipsing binary method of distance determination is very flexible and has many different approaches. We decided to use as our main approach the method based on the empirical surface brightness-color relations. In order to derive the photometric parallax to an eclipsing binary we need to calculate the individual angular diameters of the components. An angular diameter is calculated with the formula:

$$
\phi(\text { mas })=10^{0.2 \cdot\left(S-m_{0}\right)}
$$

where $S$ is the surface brightness in a given band and $m_{0}$ is the dereddened magnitude of a star in that band. In most cases $S$ is calibrated as a function of an intrinsic color of a star, usually in the form of a polynomial. The photometric parallax $\varpi_{\text {Phot }}$ follows from the equation:

$$
\varpi_{\text {Phot }}(\text { mas })=107.52 \cdot \phi(\text { mas }) / R\left(\mathcal{R}_{\odot}\right),
$$

where $R$ is the radius of the star and the conversion factor is equal to $1 \mathrm{au} / 2 \mathcal{R}_{\odot}$ assuming the solar nominal radius $\mathcal{R}_{\odot}=$ $695,700 \mathrm{~km}$ (Habbereiter et al. 2008; Mamajek et al. 2015) and a length of the astronomical unit $1 \mathrm{au}=149,597,871 \mathrm{~km}$ (Pitjeva \& Standish 2009).

We determined parallaxes using several SBC relations from the literature calibrated on infrared colors (Kervella et al. 2004; Di Benedetto 2005; Boyajian et al. 2014; Challouf et al. 2014). The most useful calibrations of $S$ are based on $(B-K)$ and $(V-K)$ colors, because the reddening vector is almost parallel to the SBC relations for intermediate- and late-type stars. Moreover, these 

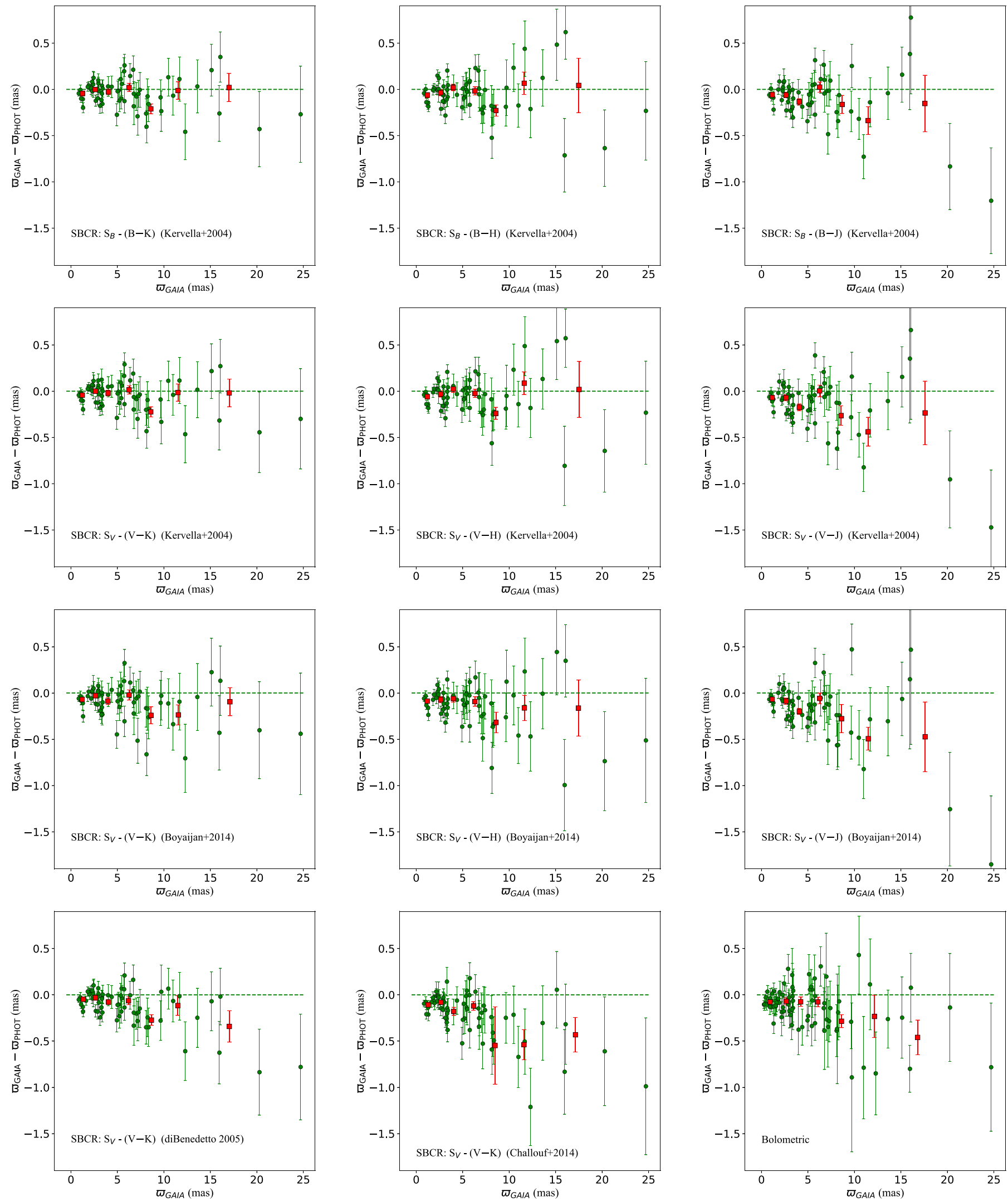

Figure 3. Individual parallax differences $d \varpi=\varpi_{\text {Gaia }}-\varpi_{\text {Phot }}$ of eclipsing binaries as a function of the Gaia parallaxes for all SBC relations used (green circles). The red squares are binned weighted means. The direct comparison of $\varpi_{\text {Gaia }}$ and $\varpi_{\text {Phot }}$ for a few SBC relations is given in Figure 5.

relations have very low intrinsic dispersion (e.g., Kervella et al. 2004; Graczyk et al. 2017). However, we also used calibrations based on other infrared colors to have a more detailed view of possible systematics. The parallax to a given eclipsing binary was calculated as the unweighted average of the individual parallaxes of its components. 

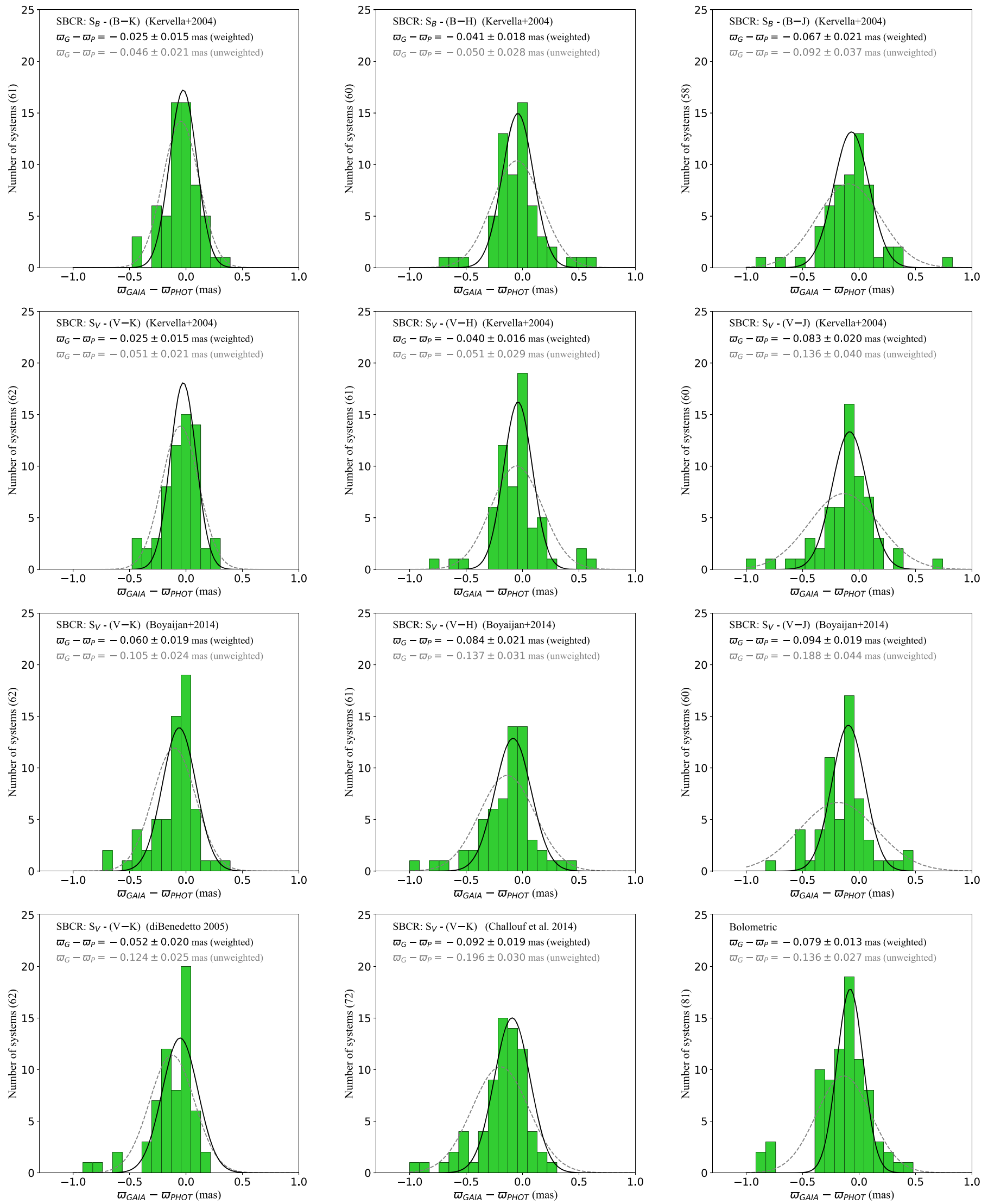

Figure 4. Histograms of the parallax differences $d \varpi=\varpi_{\text {Gaia }}-\varpi_{\text {Phot }}$ for all SBC relations used. The Gaussian distributions correspond to weighted (continuous line) and unweighted (broken line) mean values. They are given just for a reference. 
Table 5

The Zero-point Shifts $\varpi_{\text {Gaia }}-\varpi_{\text {Phot }}$ Determined with Eclipsing Binaries for the Different SBCRs

\begin{tabular}{|c|c|c|c|c|c|c|c|}
\hline \multirow{2}{*}{$\begin{array}{l}\text { SBC } \\
\text { Relation }\end{array}$} & \multirow[t]{2}{*}{ Band } & \multirow[t]{2}{*}{ Color } & \multirow{2}{*}{$\begin{array}{l}\text { Range } \\
\text { of Color }\end{array}$} & \multirow{2}{*}{$\begin{array}{l}\log g \\
(\operatorname{dex})\end{array}$} & \multirow{2}{*}{$\begin{array}{l}\text { Number of } \\
\text { Systems }\end{array}$} & \multicolumn{2}{|c|}{$\left(\varpi_{\text {Gaia }}-\varpi_{\text {Phot }}\right)$ (mas) } \\
\hline & & & & & & Unweighted & Weighted \\
\hline Kervella et al. (2004) & $B$ & $B-K$ & -0.25 to 2.36 & $>3.5$ & 61 & $-0.046 \pm 0.021$ & $-0.025 \pm 0.015$ \\
\hline Kervella et al. (2004) & $B$ & $B-J$ & -0.17 to 2.08 & $>3.5$ & 58 & $-0.092 \pm 0.037$ & $-0.067 \pm 0.021$ \\
\hline Kervella et al. (2004) & $V$ & $V-K$ & -0.20 to 1.75 & $>3.5$ & 62 & $-0.051 \pm 0.021$ & $-0.025 \pm 0.015$ \\
\hline Kervella et al. (2004) & $V$ & $V-H$ & -0.15 to 1.66 & $>3.5$ & 61 & $-0.051 \pm 0.029$ & $-0.040 \pm 0.016$ \\
\hline Kervella et al. (2004) & $V$ & $V-J$ & -0.12 to 1.47 & $>3.5$ & 60 & $-0.136 \pm 0.040$ & $-0.083 \pm 0.020$ \\
\hline Boyajian et al. (2014) & $V$ & $V-K$ & -0.15 to 1.75 & $>3.5$ & 62 & $-0.105 \pm 0.024$ & $-0.060 \pm 0.019$ \\
\hline Boyajian et al. (2014) & $V$ & $V-H$ & -0.13 to 1.66 & $>3.5$ & 61 & $-0.137 \pm 0.031$ & $-0.084 \pm 0.021$ \\
\hline Boyajian et al. (2014) & $V$ & $V-J$ & -0.12 to 1.47 & $>3.5$ & 60 & $-0.188 \pm 0.044$ & $-0.094 \pm 0.019$ \\
\hline Challouf et al. (2014) & $V$ & $V-K$ & -0.60 to 4.93 & $>2.0$ & 72 & $-0.196 \pm 0.030$ & $-0.092 \pm 0.019$ \\
\hline
\end{tabular}

Note. The blue edge of the color range is constrained by the color range validity of the SBC relation (with the exception of the relation by Challouf et al. 2014) and the red range is contrained by most red systems in the sample.

\subsection{Calculation of the Shift with Respect to Gaia Parallaxes}

We calculated individual parallax differences $d \varpi_{i}=$ $\left(\varpi_{\text {Gaia }}-\varpi_{\text {Phot }}\right)_{i}$ for every $i$ th eclipsing binary and for each $\mathrm{SBC}$ relation used. For a given SBC relation we then determined the zero-point shift between the photometric and Gaia parallaxes as unweighted and weighted means of the differences. The individual differences for all used SBC relations are presented in Figure 3. The errors of the differences were calculated taking into account systematic uncertainties of the SBC relations: $2 \%$ for calibrations by Kervella et al. (2004) and Di Benedetto (2005), 3\% for calibrations by Boyajian et al. (2014), and 3.5\% for calibration by Challouf et al. (2014) - their Equation (13). Weighted means are dominated by more distant systems for which the error on $d \varpi$ is much smaller than for nearby eclipsing binaries. This is a bit counterintuitive and it needs an explanation. Error on $d \varpi$ comes from two uncertainties: that of the Gaia parallax and that of the eclipsing binary photometric parallax. While the former for stars with $G<14$ mag (all our sample) is almost independent of the distance and equal to 0.04 mas on average (Lindegren et al. 2018), the latter decreases with the distance because it is, in the majority of cases, dominated by a systematic uncertainty of the SBCR. Thus for a systematic error of $2 \%$ on the photometric parallax the uncertainty is about 0.2 mas for a system lying $100 \mathrm{pc}$ from the Sun and about 0.02 mas for a system lying $1 \mathrm{kpc}$ away. In Figure 4 we present histograms of the differences for all the SBCR used, and also for parallaxes derived with the bolometric flux scaling method. The Gaussians correspond to a standard uncertainty distribution on weighted (continuous line) and unweighted means (dashed line).

\section{Results}

Table 5 presents a summary of the zero-point $\varpi_{\text {Gaia }}-\varpi_{\text {Phot }}$ shifts for the different SBC relations, and in Figure 5 we show exemplary relations between trigonometric Gaia DR2 and photometric, eclipsing binary parallaxes. The photometric parallaxes derived from bolometric fluxes correspond well with Gaia DR2 parallaxes, although the offset between both is clear. The amount of the offset of $-0.067 \pm 0.012$ mas is marginally consistent with the zero-point shift reported by Stassun \& Torres (2018). Our sample agrees in about $80 \%$ with a sample used by Stassun \& Torres (2018), we also adopted the same solar constants; however, we used a different method to derive $L_{\mathrm{bol}}$, which may account for the difference with their result. We point out that the accuracy of the derived offset is limited by the accuracy of the zero-points of the different methods used to determine the effective temperatures and the choice of solar constants. However, the problem of homogenization of the temperature determination for all the sample and the proper calibration of the temperature zero-point scale is beyond the scope of this paper. We therefore prefer to establish the Gaia zero-point shift solely based on the empirical SBC relations, which allows for a homogenous treatment of the sample (and its subsamples).

As pointed out already by Stassun \& Torres (2018) there is almost perfect agreement between the photometric and Gaia $\mathrm{DR} 2$ parallaxes. However, some of the $\mathrm{SBC}$ relations result in a larger offset and larger differences (e.g., Challouf et al. 2014) - see Figures 3 and 4. The possible reasons for this are discussed in the next section.

The SBC relations, which result in the smallest differences and best agreement with Gaia DR2 parallaxes are those based on $(B-K)$ and $(V-K)$ colors and calibrated by Kervella et al. (2004). They are also the relations that result in the smallest internal dispersions of the differences. Calibrations based on these two colors are the least reddening dependent because the reddening line on the surface brightness $S$-color diagram is almost parallel to the relations themselves. Moreover, the calibrations by Kervella et al. (2004) were done for mainsequence dwarfs and subgiants, which constitute the overwhelming majority of the eclipsing binary component stars in our sample. The resulting zero-point shift based on the $(B-K)$ and $(V-K)$ relations is $-0.025 \pm 0.011$ mas. Relations by Kervella et al. (2004) calibrated on the other optical-infrared colors are more reddening dependent and also show significantly larger scatter of the differences.

Another SBC relation that we used extensively for the determination of the distances to the Magellanic Clouds 

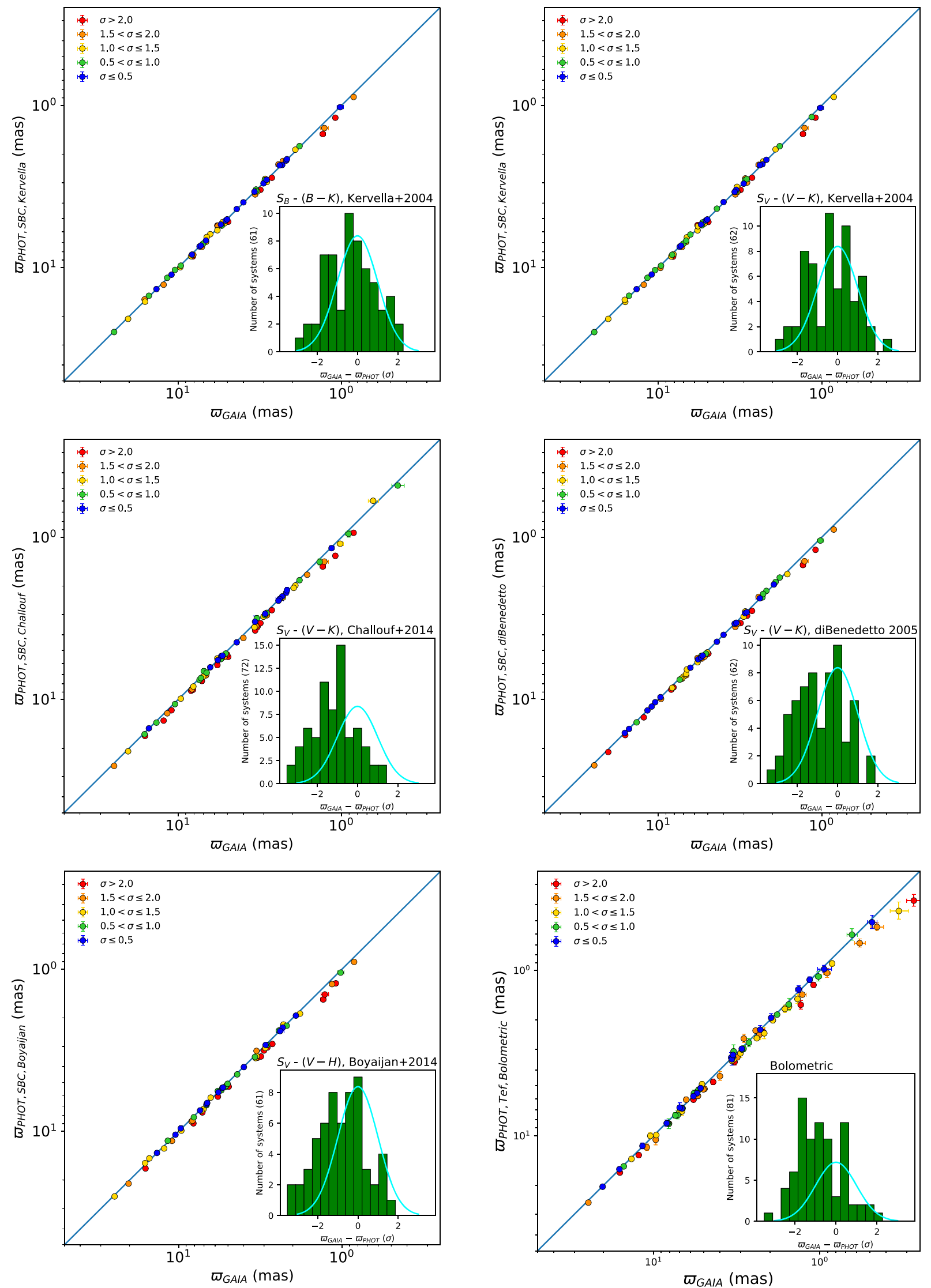

Figure 5. Direct comparison of the Gaia and the photometric parallaxes for a few SBC relations. The errorbars in most cases are smaller than the size of the symbols. Insets show histograms like in Figure 4 but expressed in terms of uncertainty of $d \varpi$. The Gaussians show the expected distribution if no offset is present. 

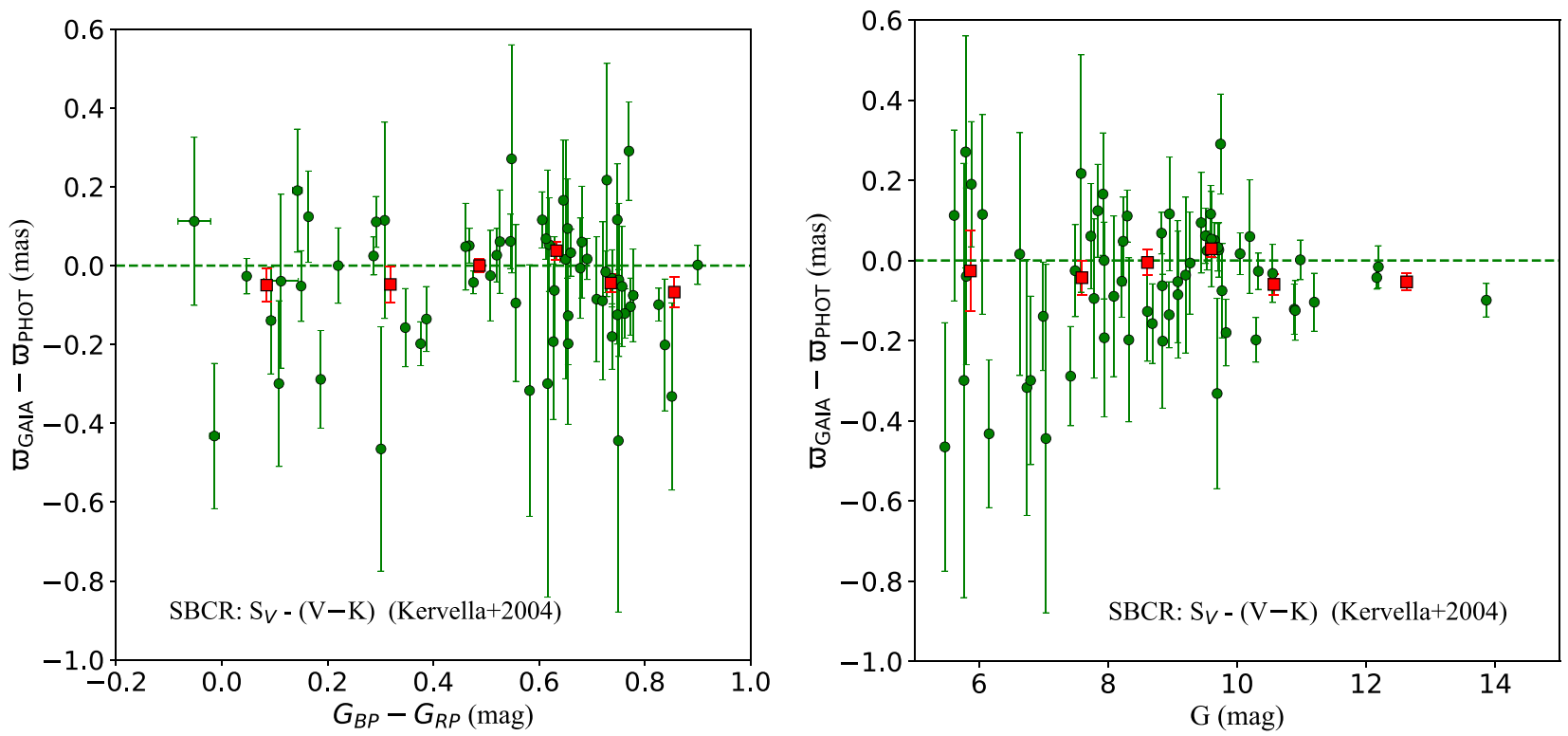

Figure 6. Color and magnitude dependency of the parallax differences for the SBC relation by Kervella et al. (2004). The meanings of the symbols are the same as in Figure 3.

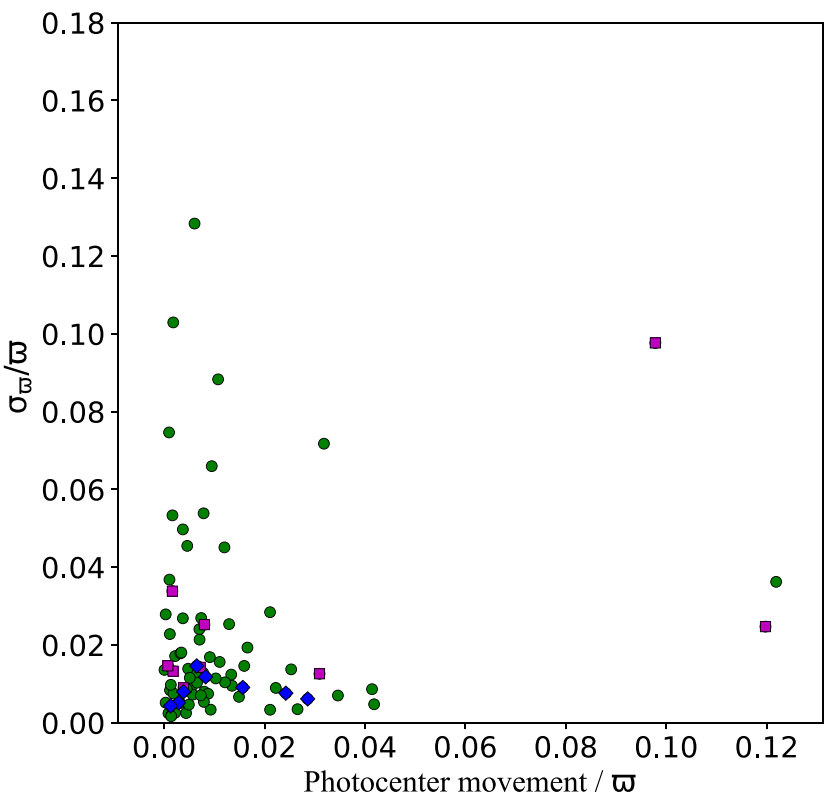

Figure 7. Photocenter movement of eclipsing binaries in our sample expressed as a fraction of Gaia DR2 parallax vs. the fractional uncertainty of the Gaia parallax. The meanings of the symbols are the same as in Figure 1.

(Pietrzyński et al. 2013; Graczyk et al. 2014) was calibrated by Di Benedetto (2005). This relation is calibrated on a mixture of giant and dwarf stars. The resulting zero-point shift is $d \varpi=-0.052 \pm 0.020$ mas and is within $1.3 \sigma$ consistent with the $d \varpi$ derived from the Kervella et al. SBC relations. Combining these two determinations we obtain a value of $d \varpi=-0.031 \pm 0.011$.

\section{Discussion}

Using the most reliable SBC relations available in the literature, we derived a zero-point shift for Gaia DR2 parallaxes of $d \varpi=-0.031 \pm 0.011$ mas. However, there are significant differences of the zero-point shifts resulting from applying (1) different SBC calibrations, and (2) different colors.

The reasons for the existence of the SBC calibration dependent zero-point shifts are somewhat unclear. A choice of different interferometric stellar angular diameter sets will result in different calibrations. Most notably a calibration derived from a mixture of all kinds of stars (e.g., Challouf et al. 2014) may lead to a systematic difference with a calibration based on a mixture of dwarf and subgiant stars only (e.g., Kervella et al. 2004). However, more worrisome is a systematic difference of the zero-point shifts derived from the same colors but from two different calibrations (Kervella et al. 2004; Boyajian et al. 2014) that are based on angular diameters of dwarf and subgiants stars and calibrated on the Johnson system. We searched relevant papers to find the differences. Kervella et al. (2004) use a small but well characterized sample of stars, while Boyajian et al. (2014) used a much larger sample but containing multiple and variable stars, and also utilizing saturated 2MASS photometry for some bright stars. Because of this we tend to prefer the former calibrations over the latter. The systematic differences between the calibrations (see Table 5) vary from -0.011 to -0.044 mas with a weighted average of -0.031 mas.

Regarding (2) there is a clear trend that relations calibrated on shorter-wavelength colors (and therefore being more reddening dependent) give systematically larger zero-point shifts and larger scatter of the differences, with the largest shifts coming from SBC relation calibrations based on the $(V-J)$ color. We tried to remove the shifts by a rescaling of all individual reddening estimates according to $E(B-V)_{\text {new }}=$ $C \cdot E(B-V)$, where $C>1$ is a scaling factor. Unless $C$ is larger then about 4 there is no possibility to obtain an agreement between zero-point shifts derived from different colors. But such a large value of the scaling factor would lead to an unrealistically large extinction for most of the targets in our sample, and would be strongly at odds with independent reddening determinations by Stassun \& Torres (2016). 
Another, but less likely, possibility is that the transformation equations from Section 2.1.2 contain some systematic error leading to color dependent zero-point shifts. And at last the SBC relations calibrated on colors containing $J$ and $H$ may be problematic by themselves because of a proper calibration of the ground-based photometry in these filters is difficult (strong and variable atmospheric extinction) and because of stronger sensibility to the interstellar reddening.

We investigated how the global shift depends on a distance. We binned up individual $d \varpi_{i}$ in several bins-see Figure 3; however, no clear systematic trend can be noticed. We also investigated a possible Gaia color $G_{B P}-G_{R P}$ and $G$ magnitude dependency. We plotted $d \varpi$ differences for one specific SBC relation-Figure 6. As previously we do not detect any systematic trend. We also investigated the correlation of $d \varpi$ with sky position. We calculated separately shifts for equatorial and ecliptic hemispheres. The differences between hemispheres are in both cases within $1 \sigma$ uncertainty and thus not statistically significant.

The comparison with previous determinations of $d \varpi$ shows excellent agreement with the Gaia Team results (Arenou et al. 2018; Lindegren et al. 2018). In fact we confirm here the zeropoint shift of $d \varpi=-0.029$ mas reported by the Gaia Team. However, comparisons with other independent determinations show some discrepancies. Although the $d \varpi$ value reported by Riess et al. (2018) agrees with our value at the $1 \sigma$ level, the zero-point shifts reported by Stassun \& Torres (2018) and Zinn et al. (2018) are somewhat discrepant. The problem with the shift reported by Zinn et al. (2018) is its unrealistically small systematic uncertainty, which ignores systematics of asteroseismic relations themselves and the zero-point uncertainty of the temperature scale used by the authors. Because of this a comparison of the shift by Zinn et al. (2018) with other reported shifts is vague.

Of special interest is the knowledge of how binarity will impact Gaia parallaxes, and the determination of the global zero-point shift. In order to quantify this, we calculated the photocenter movement for our $\sim 80$ eclipsing binaries and compared them with the uncertainties on Gaia DR2 parallaxes - see Figure 7. For the overwhelming majority of the systems the photocenter movement is only a very small fraction of the parallax itself. Only in two systems could Gaia DR2 probably recognize the photocenter movement (EPIC 211409263 and V380 Cyg). Thus we can consider the Gaia DR2 parallaxes of the eclipsing binaries in our sample as practically unaffected by binarity.

We dedicate this paper to our colleague Zbigniew ("Zibi”) Kołaczkowski who passed away much too early.

We thank the anonymous referee for valuable comments and remarks on the manuscript. The research leading to these results has received funding from the European Research Council (ERC) under the European Union's Horizon 2020 research and innovation program (grant agreement No. 695099).

We are grateful for financial support from Polish National Science Center grant MAESTRO 2012/06/A/ST9/00269. Support from the BASAL Centro de Astrofísica y Tecnologías Afines (CATA) grant AFB-170002, the Millenium Institute of Astrophysics (MAS) of the Iniciativa Cientifica Milenio del Ministerio de Economia, Fomento y Turismo de Chile, project IC120009 and the IdP II 2015000264 grant of the Polish
Ministry of Science and Higher Education is also acknowledged. We are also thankful to the staff in La Silla Observatory (ESO) and Las Campanas Observatory (Carnegie) for their excellent support.

This work has made use of data from the European Space Agency (ESA) mission Gaia (https://www.cosmos.esa.int/ gaia), processed by the Gaia Data Processing and Analysis Consortium (DPAC, https://www.cosmos.esa.int/web/gaia/ dpac/consortium). Funding for the DPAC has been provided by national institutions, in particular, the institutions participating in the Gaia Multilateral Agreement.

This research has made extensive use of the excellent astronomical SIMBAD database and of the VizieR catalog access tool, operated at CDS, Strasbourg, France, and made also use of NASA's Astrophysics Data System Bibliographic Services (ADS).

This publication makes use of data products from the Two Micron All Sky Survey, which is a joint project of the University of Massachusetts and the Infrared Processing and Analysis Center/California Institute of Technology, funded by the National Aeronautics and Space Administration and the National Science Foundation.

\section{ORCID iDs}

Dariusz Graczyk (1) https://orcid.org/0000-0002-7355-9775 Wolfgang Gieren (iD https://orcid.org/0000-0003-1405-9954 Jesper Storm (1) https://orcid.org/0000-0002-8627-6096 Nicolas Nardetto (iD https://orcid.org/0000-0002-7399-0231 Alexandre Gallenne (iD https://orcid.org/0000-0001-7853-4094 Pierre F. L. Maxted (1D https://orcid.org/0000-0003-3794-1317 Pierre Kervella (iD https://orcid.org/0000-0003-0626-1749 Bogumił Pilecki (ib https://orcid.org/0000-0003-3861-8124 Paulina Karczmarek (iD https://orcid.org/0000-00020136-0046

Mónica Taormina (iD https://orcid.org/0000-0002-1560-8620

Piotr Wielgórski (iD https://orcid.org/0000-0002-1662-5756

Radosław Smolec (iD https://orcid.org/0000-0001-7217-4884

\section{References}

Albrecht, S., Reffert, S., Snellen, I., Quirrenbach, A., \& Mitchell, D. S. 2007, A\&A, 474, 565

Albrecht, S., Reffert, S., Snellen, I. A. G., \& Winn, J. N. 2009, Natur, 461, 373

Albrecht, S., Sewiatan, J., Torres, G., Fabrycky, D. C., \& Winn, J. N. 2013, ApJ, 767, 32

Albrecht, S., Winn, J. N., Torres, G., et al. 2014, ApJ, 785, 83

Andersen, J. 1975, A\&A, 44, 355

Andersen, J., \& Clausen, J. V. 1989, A\&A, 213, 183

Andersen, J., Clausen, J. V., \& Giménez, A. 1993, A\&A, 277, 439

Andersen, J., Clausen, J. V., \& Magain, P. 1989, A\&A, 211, 346

Andersen, J., Clausen, J. V., \& Nordstrom, B. 1984, A\&A, 137, 281

Andersen, J., Clausen, J. V., \& Nordström, B. 1987a, A\&A, 175, 60

Andersen, J., Clausen, J. V., Nordstrom, B., \& Popper, D. M. 1985, A\&A, 151,329

Andersen, J., Clausen, J. V., Nordström, B., \& Reipurth, B. 1983, A\&A, 121,271

Andersen, J., Clausen, J. V., Nordström, B., Tomkin, J., \& Mayor, M. 1991, A\&A, 246, 99

Andersen, J., García, J. M., Giménez, A., \& Nordström, B. 1987b, A\&A, 174,107

Andersen, J., Gjerløff, H., \& Imbert, M. 1975, A\&A, 44, 349

Andersen, J., \& Vaz, L. P. R. 1984, A\&A, 130, 102

Arenou, F., Luri, X., Babusiaux, C., et al. 2018, A\&A, 616, 17

Bailer-Jones, C. A. L. 2015, PASP, 127, 994

Bailer-Jones, C. A. L., Rybizky, J., Fouesneau, M., Mantelet, G., \& Andrae, R. 2018, AJ, 156, 58

Bakiş, V., Bakiş, H., Bilir, S., \& Eker, Z. 2016, PASA, 33, 46 
Bakiş, V., Bakiş, H., Demircan, O., \& Eker, Z. 2008, MNRAS, 384, 1657 Barembaum, M. J., \& Etzel, P. B. 1995, AJ, 109, 2680

Bessell, M. 2000, PASP, 112, 961

Bessell, M. S., \& Brett, J. M. 1988, PASP, 100, 1134

Bilir, S., Ak, T., Soydugan, E., et al. 2008, AN, 329, 835

Boyajian, T. S., van Belle, G., \& von Braun, K. 2014, AJ, 147, 47

Budding, E., Butland, R., \& Blackford, M. 2015, MNRAS, 448, 3784

Capitanio, L., Lallement, R., Vergely, J. L., Elyajouri, M., \& Monreal-Ibero, A. 2017, A\&A, 606, 65

Carpenter, J. M. 2001, AJ, 121, 2851

Casagrande, L., Portinari, L., Glass, I. S., et al. 2014, MNRAS, 439, 2060

Challouf, M., Nardetto, N., Mourard, D., et al. 2014, A\&A, 570, 104

Clausen, J. V. 1991, A\&A, 246, 397

Clausen, J. V., Bruntt, H., Olsen., E. H., Helt, B. E., \& Claret, A. 2010, A\&A, 511,22

Clausen, J. V., Frandsen, S., Bruntt, H., et al. 2010, A\&A, 516, 42

Clausen, J. V., Giménez, A., \& Scarfe, C. 1986, A\&A, 167, 287

Clausen, J. V., Helt, B. E., Gimenez, A., et al. 2007, A\&A, 461, 1065

Clausen, J. V., Helt, B. E., \& Olsen, E. H. 2001, A\&A, 374, 980

Clausen, J. V., Torres, G., Bruntt, H., et al. 2008, A\&A, 487, 1095

David, T. J., Conroy, K. E., Hillenbrand, L. A., et al. 2016, AJ, 151, 112

Demircan, O., Kaya, Y., \& Tufekcioglu, Z. 1994, Ap\&SS, 222, 213

Di Benedetto, G. P. 2005, MNRAS, 357, 174

Eker, Z., Bilir, S., Soydugan, F., et al. 2014, PASA, 31, 24

Fekel, F. C., Williamson, M. H., \& Henry, G. W. 2011, AJ, 141, 145

Fitzpatrick, E. L., \& Massa, D. 2007, ApJ, 663, 320

Flower, P. J. 1996, ApJ, 469, 355

Gaia Collaboration, Brown, A. G. A., Vallenari, A., Prusti, T., et al. 2018, A\&A, 616,

Gaia Collaboration, Prusti, T., de Bruijne, J. H. J., Brown, A. G. A., et al. 2016, A\&A, 595, 1

Gallenne, A., Pietrzyński, G., Graczyk, D., et al. 2016, A\&A, 586, 35

Giuricin, G., Mardirossian, F., Mezzetti, M., \& Predolin, F. 1980, A\&A, 85, 259

Graczyk, D., Konorski, P., Pietrzyński, G., et al. 2017, ApJ, 837, 7

Graczyk, D., Pietrzyński, G., Thompson, I. B., et al. 2014, ApJ, 780, 59

Graczyk, D., Smolec, R., Pavlovski, K., et al. 2016, A\&A, 594, 92

Griffin, R. F. 2013, Obs, 133, 156

Groenewegen, M. A. T. 2018, arXiv:1808.05796

Groenewegen, M. A. T., Decin, L., Salaris, M., \& De Cat, P. 2007, A\&A, 463,579

Gronbech, B., Gyldenkerne, K., \& Jorgensen, H. E. 1977, A\&A, 55, 401

Habbereiter, M., Schmutz, W., \& Kosovichev, A. G. 2008, ApJ, 675, 53

Harmanec, P., Holmgren, D. E., Wolf, M., et al. 2014, A\&A, 563, 120

Hełminiak, K. G., Konacki, M., Ratajczak, M., \& Muterspaugh, M. W. 2009, MNRAS, 400, 969

Hełminiak, K. G., Ukita, N., Kambe, E., \& Konacki, M. 2015, ApJ, 813, 25

Henry, G. W., Fekel, F. C., Sowell, J. R., \& Gearhart, J. S. 2006, AJ, 132, 2489

Høg, E., Fabricius, C., Makarov, V. V., et al. 2000, A\&A, 357, 367

Çakirli, Ö, Ibanoğlu, C., Bilir, S., \& Sipahi, E. 2009, MNRAS, 395, 1649

Imbert, M. 1985, A\&AS, 59, 357

Imbert, M. 1986, A\&AS, 65, 97

Imbert, M. 1987, A\&AS, 69, 397

Imbert, M. 2002, A\&A, 387, 850

Kervella, P., Arenou, F., Mignard, F., \& Thevenin, F. 2019, A\&A, in press (arXiv:1811.08902)

Kervella, P., Thévenin, F., Di Folco, E., \& Ségransan, D. 2004, A\&A, 426, 297 Khaliullin, K. F., Khaliullina, A. I., \& Krylov, A. V. 2001, ARep, 45, 888

Kirkby-Knet, J. A., Maxted, P. F. L., Serenelli, A. M., et al. 2018, A\&A, 615, 135 Kurucz, R. 1993, ATLAS9 Stellar Atmosphere Programs and $2 \mathrm{~km} \mathrm{~s}^{-1}$ Grid, Kurucz CD-ROM No. 13 (Cambridge, MA: SAO)

Lacy, C. H. 1987, AJ, 94, 1670

Lacy, C. H., \& Frueh, M. L. 1985, ApJ, 295, 569

Lacy, C. H., \& Frueh, M. L. 1987, ApJ, 94, 712

Lacy, C. H., Gülmen, O., Güdür, N., \& Sezer, C. 1989, AJ, 97, 822

Lacy, C. H. S. 1997, AJ, 113, 1406

Lacy, C. H. S. 2002, AJ, 124, 1162

Lacy, C. H. S., Claret, A., \& Sabby, J. A. 2004a, AJ, 128, 1340

Lacy, C. H. S., Claret, A., \& Sabby, J. A. 2004b, AJ, 128, 1840

Lacy, C. H. S., Claret, A., Sabby, J. A., Hood, B., \& Secosan, F. 2004c, AJ, 128,3005

Lacy, C. H. S., \& Fekel, F. C. 2011, AJ, 142, 185

Lacy, C. H. S., \& Fekel, F. C. 2014, AJ, 148, 71

Lacy, C. H. S., Fekel, F. C., \& Claret, A. 2012, AJ, 144, 63

Lacy, C. H. S., Torres, G., Claret, A., et al. 2000, AJ, 119, 1389

Lacy, C. H. S., Torres, G., \& Claret, A. 2008, AJ, 135, 1757

Lacy, C. H. S., Torres, G., Claret, A., \& Menke, J. L. 2006, AJ, 131, 2664
Lacy, C. H. S., Torres, G., Fekel, F. C., \& Muterspaugh, M. W. 2014, AJ, 147, 148

Lacy, C. H. S., Torres, G., Fekel, F. C., Sabby, J. A., \& Claret, A. 2012, AJ, 143,129

Lallement, R., Vergely, J.-L., Valette, B., et al. 2014, A\&A, 561, 91

Latham, D. W., Nordstroem, B., Andersen, J., et al. 1996, A\&A, 314, 864

Lester, K. V., \& Gies, D. R. 2018, AJ, 156, 8

Lindegren, L., Hernández, J., Bombrun, A., et al. 2018, A\&A, 616, 2

Mamajek, E. E., Prsa, A., Torres, G., et al. 2015, IAU 2015 Resolution B3, arXiv: 1510.07674

Maxted, P. F. L., Hutcheon, R. J., Torres, G., et al. 2015, A\&A, 578, 25

Mermilliod, J. C. 1997, yCat, 2168, 0

Munari, U., Dallaporta, S., Siviero, A., et al. 2004, A\&A, 418, L31

Muraveva, T., Delgado, H. E., Clementini, G., Sarro, L. M., \& Garofalo, A. 2018, arXiv:1805.08742

North, P., Studer, M., \& Kunzli, M. 1997, A\&A, 324, 137

Ochsenbein, F., Bauer, P., \& Marcout, J. 2000, A\&AS, 143, 23

Pavlovski, K., Southworth, J., Kolbas, V., \& Smalley, B. 2014, MNRAS, 438, 590

Pavlovski, K., Tamajo, E., Koubský, P., et al. 2009, MNRAS, 400, 791

Pietrzyński, G., Graczyk, D., Gieren, W., et al. 2013, Natur, 495, 76

Pitjeva, E. V., \& Standish, E. M. 2009, CeMDA, 103, 356

Popper, D. M. 1971, ApJ, 169, 549

Popper, D. M. 1982, ApJ, 254, 203

Popper, D. M. 1983, AJ, 88, 1242

Popper, D. M. 1987, AJ, 93, 672

Popper, D. M. 1998, AJ, 115, 338

Popper, D. M., Andersen, J., Clausen, J. V., \& Nordstrom, B. 1985, AJ, 90, 1324

Popper, D. M., Lacy, C. H., Frueh, M. L., \& Turner, A. E. 1986, AJ, 91, 383

Pribulla, T., Merand, A., Kervella, P., et al. 2018, A\&A, 616, 49

Ratajczak, M., Kwiatkowski, T., Schwarzenberg-Czerny, A., et al. 2010, MNRAS, 402, 2424

Rawls, M. L., Gaulme, P., McKeever, J., et al. 2016, ApJ, 818, 108

Ribas, I., Jordi, C., \& Jordi, T. 1999, MNRAS, 309, 199

Riess, A. G., Casertano, S., Yuan, W., et al. 2018, ApJ, 861, 126

Sabby, J. A., Lacy, C. H. S., Ibanoglu, C., \& Claret, A. 2011, AJ, 141, 195

Sandquist, E. L., Jessen-Hansen, J., Shetrone, M. D., et al. 2016, ApJ, 831, 11

Sandquist, E. L., Mathieu, R. D., Quinn, S. N., et al. 2018, AJ, 155, 152

Schlegel, D. J., Finkbeiner, D. P., \& Davis, M. 1998, ApJ, 500, 525

Skrutskie, M. F., Cutri, R. M., Stiening, R., et al. 2006, AJ, 131, 1163

Southworth, J. 2013, A\&A, 557, 119

Southworth, J. 2015, in ASP Conf. Ser. 496, Living Together: Planets, Host Stars and Binaries, ed. S. Rucinski, G. Torres, \& M. Zejda (San Francisco, CA: ASP), 164

Southworth, J., \& Clausen, J. V. 2007, A\&A, 461, 1077

Southworth, J., Maxted, P. F. L., \& Smalley, B. 2005, A\&A, 429, 645

Southworth, J., Pavlovski, K., Tamajo, E., et al. 2011, MNRAS, 414, 3740

Southworth, J., Smalley, B., Maxted, P. F. L., Claret, A., \& Etzel, P. B. 2005, MNRAS, 363, 529

Sowell, J. R., Henry, G. W., \& Fekel, F. C. 2012, AJ, 143, 5

Stassun, K. G., \& Torres, G. 2016, AJ, 152, 180

Stassun, K. G., \& Torres, G. 2018, ApJ, 862, 61

Stickland, D. J., Lloyd, C., \& Corcoran, M. F. 1994, Obs, 114, 284

Suchomska, K., Graczyk, D., Smolec, R., et al. 2015, MNRAS, 451, 651

Tkachenko, A., Degroote, P., Aerts, C., et al. 2014, MNRAS, 438, 3093

Tomasella, L., Munari, U., Cassisi, S., et al. 2008a, A\&A, 483, 263

Tomasella, L., Munari, U., Siviero, A, et al. 2008b, A\&A, 480, 465

Tomkin, J., \& Fekel, F. C. 2006, AJ, 131, 2652

Torres, G., Andersen, J., \& Giménez, A. 2010, A\&ARv, 18, 67

Torres, G., Andersen, J., Nordström, B., \& Latham, D. W. 2000, AJ, 119, 1942

Torres, G., \& Lacy, C. H. S. 2009, AJ, 137, 507

Torres, G., Lacy, C. H. S., Claret, A., et al. 1999, AJ, 118, 1831

Torres, G., Lacy, C. H. S., \& Claret, A. 2009, AJ, 138, 1622

Torres, G., Lacy, C. H. S., Pavlovski, K., et al. 2014, ApJ, 797, 31

Torres, G., Lacy, C. H. S., Pavlovski, K., Fekel, F. C., \& Muterspaugh, M. W 2015, AJ, 150, 154

Torres, G., McGruder, C. D., Siverd, R. J., et al. 2017, ApJ, 836, 177

van Hamme, W., \& Wilson, R. E. 2007, ApJ, 661, 1129

Veramendi, M. E., \& González, J. F. 2015, NewA, 34, 266

Vos, J., Clausen, J. V., Jørgensen, U. G., et al. 2012, A\&A, 540, 64

Walker, R. L., \& Chambliss, C. R. 1983, AJ, 88, 535

Williamon, R. M., Sowell, J. R., \& van Hamme, W. 2004, AJ, 128, 1319

Wilson, R. E. 1979, ApJ, 234, 1054

Wilson, R. E. 1990, ApJ, 356, 613

Wilson, R. E., \& Devinney, E. J. 1971, ApJ, 166, 605

Worthey, G., \& Lee, H. 2011, ApJS, 193, 1

Yakut, K., Aerts, C., \& Morel, T. 2007, A\&A, 467, 647

Zinn, J. C., Pinsonneault, M. H., Huber, D., \& Stello, D. 2018, arXiv:1805.02650 